\title{
Detection of partial-thickness supraspinatus tendon tears: is a single direct MR arthrography series in ABER position as accurate as conventional MR arthrography?
}

\author{
Saskia A. Schreinemachers • \\ Victor P. M. van der Hulst • W. Jaap Willems • \\ Shandra Bipat $\cdot$ Henk-Jan van der Woude
}

Received: 31 December 2008 /Revised: 23 February 2009/Accepted: 1 March 2009/Published online: 18 March 2009

(C) The Author(s) 2009. This article is published with open access at Springerlink.com

\begin{abstract}
Purpose The purpose of this study was to retrospectively evaluate sensitivity and specificity of a single magnetic resonance (MR) arthrography series in abduction external rotation (ABER) position compared with conventional MR arthrography for detection of supraspinatus tendon tears, with arthroscopy as gold standard, and to assess interobserver variability.

Materials and methods Institutional review board approval was obtained; informed consent was waived. MR arthrograms of 250 patients (170 men and 80 women; mean age, 36 years) were retrospectively and independently evaluated by three observers. Oblique coronal T1-weighted fatsuppressed images, proton density, and T2-weighted images and axial T1-weighted images and oblique sagittal T1weighted fat-suppressed images were analyzed to detect
\end{abstract}

No financial relationship to disclose.

S. A. Schreinemachers - V. P. M. van der Hulst

H.-J. van der Woude

Department of Radiology, Onze Lieve Vrouwe Gasthuis,

Oosterpark 9, Postbus 95500, 1090 HM Amsterdam,

The Netherlands

W. J. Willems

Orthopaedic Surgery, Onze Lieve Vrouwe Gasthuis,

Oosterpark 9, Postbus 95500, 1090 HM Amsterdam,

The Netherlands

S. Bipat

Department of Radiology, Academic Medical Center,

University of Amsterdam,

Amsterdam, The Netherlands

S. A. Schreinemachers $(\square)$

Onze Lieve Vrouwe Gasthuis,

Oosterpark 9, Postbus 95500, 1090 Amsterdam, The Netherlands

e-mail: sschreinemachers@gmail.com supraspinatus tendon tears. Separately, a single T1weighted fat-suppressed oblique axial series in ABER position was evaluated. Both protocols were scored randomly without knowledge of patients' clinical history and arthroscopy results. Tears were subclassified, based on articular surface integrity and extension (Lee classification). Interobserver agreement was assessed by kappa statistics for all patients. Ninety-two of 250 patients underwent arthroscopy; sensitivity and specificity of ABER and conventional MR arthrography were calculated and compared using paired McNemar test.

Results Weighted kappa values of ABER and conventional MR arthrography were $0.48-0.65$ and $0.60-0.67$, respectively. According to arthroscopy, 69 of 92 patients had an intact cuff, and 23 patients had a cuff tear (16 partial thickness and seven full thickness). There were no statistically significant differences between ABER and conventional MR arthrography regarding sensitivity (48$61 \%$ and $52-70 \%$, respectively) and specificity (80-94\% and $91-95 \%$ ).

Conclusion Sensitivity and specificity of a single T1weighted series in ABER position and conventional MR arthrography are comparable for assessment of rotator cuff tears.

Keywords Shoulder joint · Magnetic resonance imaging · Arthrography $\cdot$ ABER $\cdot$ Rotator cuff

\section{Introduction}

The cumulative incidence of shoulder complaints in general practice is estimated to be 11.2/1,000 patients/year, with a peak age of 45-64 years [1]. Rotator cuff tears are a 
common cause of persistent shoulder pain [1] and are often multifactorial in etiology caused by intrinsic (degeneration [2], vascularity [3]), and extrinsic factors (impingement, anterior shoulder instability, and (micro)trauma [4-6]).

The majority of tears occur in the supraspinatus tendon (up to $89 \%$ of partial thickness and $47 \%$ of full-thickness rotator cuff tears) [7]. The supraspinatus tendon is relatively hypovascular and thin at the articular side and therefore rotator cuff tears usually originate at the articular side [8]. Articular-sided partial-thickness tears are clinically two to three times more common than bursal-sided tears $[9,10]$.

The identification of partial thickness tears is important because even small tears can be a source of persistent but surgically treatable shoulder pain [11]. Furthermore, partial tears may progress to complete tears. Generally, partialthickness cuff tears of more than $50 \%$ width are treated either by repair or by extending the partial-thickness cuff tear to a full-thickness tear and by reinsertion of the tendon. The severity of partial supraspinatus tendon tears can be classified according to their location (articular, bursal, or intratendinous) and their depth [12]. Usually, the impingement related cuff tear is horizontal in configuration. The characteristics of the horizontal component of a partial thickness tear have been determined by Lee et al. [13]. Lee classified horizontal partial-thickness tears as type I (without articular surface abnormality), type II (with irregularity of the surface), and type III (flap tear of the surface) lesions.

Magnetic resonance (MR) arthrography, with joint distension using intra-articular diluted gadolinium, is considered to be the most accurate imaging technique for evaluation of the rotator cuff, with high accuracy for diagnosing of full-thickness tears (96-98\% sensitivity, 99$100 \%$ specificity $[14,15])$, and for the detection of partial thickness tears (80\% sensitivity, 97\% specificity) [14].

Preliminary studies showed that additional imaging in abduction and external rotation (ABER) position of the shoulder improves visualization of articular surface tears because of separation of the undersurface of the supraspinatus tendon from the humerus and of the horizontal component of supraspinatus tendon tears $[13,16]$.
The purpose of this study was to retrospectively evaluate sensitivity and specificity of single MR arthrography series in ABER position compared with conventional MR arthrography for the detection of supraspinatus tendon tears, with arthroscopy as gold standard, and to assess interobserver variability.

\section{Materials and methods}

\section{Patients}

This retrospective study was approved by the local medical ethics committee, and patient informed consent was waived. Two hundred fifty consecutive patients who underwent MR arthrography of the shoulder at our institution between January 2001 and July 2003 were included. Patients were included when a complete examination was available, including an oblique coronal T1weighted fat-suppressed, proton-density, and T2-weighted sequences; axial T1-weighted images, oblique sagittal T1weighted fat-suppressed images (conventional MR arthrography); and an oblique axial fat suppressed T1-weighted sequence in ABER position (ABER). The medical records were reviewed to identify the reason for referral (Table 1).

Imaging technique

All patients underwent intra-articular administration of $10 \mathrm{~mL}$ omnipaque 300 (300 mg I/ml iohexol; GE Healthcare $\mathrm{BV}$, Eindhoven) and a $10 \mathrm{~mL}$ mixture of $0.5 \mathrm{~mL}$ omniscan (0.5 mmol/ml Gd-DTPA-BMA; GE Healthcare $\mathrm{BV}$ ) added to $100 \mathrm{~mL}$ saline $0.9 \%$. Of this solution, $12-$ $15 \mathrm{cc}$ was administered after inserting an 18-gauge needle in the glenohumeral joint with fluoroscopic guidance, using either an anterior or a posterior approach. MR imaging was obtained within $30 \mathrm{~min}$ (after injection, patients were instructed to immobilize the shoulder of interest until the MR examination).

Imaging was performed with either a $1.0 \mathrm{~T}$ unit (MR Systems NT Release 4.5; Philips Medical Systems, Best,

Table 1 Baseline patient characteristics

\begin{tabular}{lll}
\hline & $250 \mathrm{MR}$ arthrograms & Study group (92 MR arthrograms) \\
\hline Male/female & $170 / 80$ & $61 / 31$ \\
Age in years: mean \pm SD (range) & $35.59(12.82)$ & $33.97(12.45)$ \\
Clinical signs/symptoms & & $28(30 \%)$ \\
Impingement & $64(25,5 \%)$ & $58(63 \%)$ \\
Instability & $134(53,5 \%)$ & $6(7 \%)$ \\
Pain of unknown origin & $52(21 \%)$ & $8(9 \%)$ \\
Previously surgery to the symptomatic shoulder & $14(6 \%)$ & $(9 \%)$ \\
\hline
\end{tabular}


The Netherlands) or $1.5 \mathrm{~T}$ unit (MR Systems Intera, Release 9.0, Philips Medical Systems).

The following imaging protocol in neutral position (conventional MR arthrography) was used: T1-weighted fast field echo [381.5-400/9.21-13.8 ms repetition time (RT)/echo time (TE), 4-mm section thickness, 0.4 gap] in the axial plane; proton density turbo spin echo (TSE) $(2,000 / 15-30 \mathrm{~ms} \mathrm{TR/}$ TE, 3-4-mm section thickness, 0.3-0.4 gap), T2-weighted TSE (3021.8-3058/90 ms TR/TE, 3-4-mm section thickness, 0.3-0.4 gap), and T1-weighted fat-suppressed TSE (500/16 TR/TE, 4-mm section thickness, 0.4 gap) in the oblique coronal plane; and T1-weighted fat-suppressed TSE (500/14 $16 \mathrm{~ms}$ TR/TE, 3-4-mm section thickness, 0.3-0.4 gap) in the oblique sagittal plane. All sequences were acquired with a NSA $2-4,256 \times 256$ matrix and $120 / 160 \times 75 / 100 \mathrm{~mm}$ field of view. A C3/C1 surface coil (1.0 T) or a Synergy Flex-M coil $(1.5 \mathrm{~T})$ was positioned on the shoulder. Total scan time was $30 \mathrm{~min}$, including positioning.

To obtain the ABER position [4] the ipsilateral hand was positioned posteriorly to the head with the elbow positioned toward the table. The surface coil was repositioned accordingly.

T1-weighted fat suppressed TSE (500-525/14-17 TR/ TE, 3-mm section thickness, 0.3 gap, $120 / 160 \times 100$ field of view, $256 \times 256$ matrix and two to three signals acquired) in an oblique axial plane. Additional scan time was $15 \mathrm{~min}$, including repositioning.

Image analysis

For image analysis, all ABER series were separated from the images in neutral position and were placed in random order. This was done by one investigator (S.A.S.) who was not involved in the image interpretation.
Two musculoskeletal radiologists (reviewer 1, H.J.W., and reviewer 2, V.P.M.H.) with at least 12 years of experience in MRI of the musculoskeletal system and one orthopedic surgeon (reviewer 3, W.J.W.) with 12 years of experience on shoulder MR arthrography, independently reviewed the MR arthrography studies in random order. The reviewers were blinded to the patient's personal details, clinical history, and symptoms and, if any, the arthroscopic results.

Presence or absence of cuff lesions was determined by each reviewer. Suspected supraspinatus tendon lesions were classified as partial-thickness tears according to the subclassification previously proposed by Lee et al. [13], (Fig. 1). Accordingly, this classification was reflected to the combined images of the MRa:

Type A: Horizontal partial-thickness tear without an articular surface abnormality

Type B: Horizontal partial-thickness tear with irregularity of the articular surface

Type C: Horizontal partial-thickness tear with a torn edge of the articular surface (flap tear)

The vertical thickness of the horizontal component of the partial thickness tears was not obtained in this study.

Full-thickness tears, represented by discontinuity of the tendon with or without presence of fluid in the subacromialsubdeltoid space, were also registered within the type $\mathrm{C}$ subgroup.

\section{Reference standard}

To determine the accuracy of MR arthrography for both conventional MR arthrography and ABER series, the
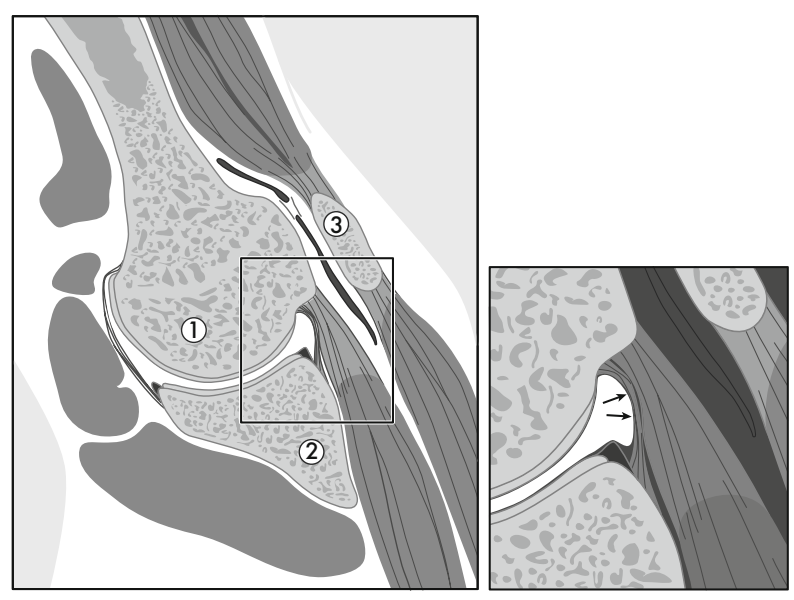

Fig. 1 Classification of the horizontal component of partial-thickness supraspinatus tendon tears according to Lee et al. [13], based on the ABER position. Schematic illustration of the anatomy of the glenohumeral joint in ABER position. 1 humeral head, 2 glenoid, 3 acromion. Detail illustrations (a-d) demonstrate: a intact supra-
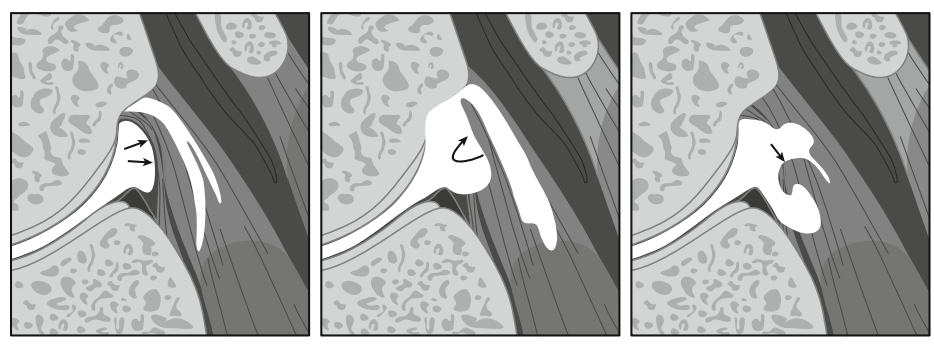

spinatus tendon (arrows). b-d Classification of the horizontal component of partial-thickness tears of the supraspinatus tendon: b tear without articular surface abnormality (type A), c tear with irregularity of the articular surface (type B), and $\mathbf{d}$ tear with torn edge of the articular surface (type C) 
patients who underwent an arthroscopy within a maximum time interval of 12 months between MR and arthroscopy were identified. Of 250 patients, 92 underwent arthroscopy within this interval. The surgical records of these 92 patients were reviewed by one investigator (S.A.S.) — who was not involved in the image interpretation for this study-to identify the arthroscopic results.

Shoulder arthroscopies were performed in lateral decubitus position under general anesthesia by two orthopedic surgeons. Routinely, two portals were used to enable a thorough arthroscopic evaluation of the glenohumeral joint and subacromial space. In case of surgery (instability repair, subacromial decompression, or cuff repair), additional portals were used.

At arthroscopy cuff lesions were classified as partial thickness (including desinsertion of the supraspinatus tendon from the footprint extending into the tendoneous tissue medially) or full-thickness tear.

Statistical analysis

\section{Interobserver variability}

The interobserver agreement was assessed by kappa statistics, and agreements in percentages were calculated for all 250 patients.

\section{Diagnostic accuracy}

Imaging findings at MR arthrography (ABER and conventional MR arthrography) and arthroscopic results were compared in 92 of 250 patients the for calculation of sensitivity and specificity. Agreement between MR arthrographic and arthroscopic registration of the presence or absence of supraspinatus tendon lesions was obtained with a sensitivity and specificity. Sensitivity and specificity of the ABER and conventional MR arthrography were compared using the paired McNemar test; a $p$ value $<0.05$ was considered a statistically significant difference.

All analyses were performed with SPSS 12.0.1 software (SPSS, Chicago, IL, USA).

\section{Results}

Patients

Of the 92 patients, 58 had been referred for instability, 28 for clinical signs of impingement, and six patients for pain of unknown origin. Eight patients had undergone surgery to the symptomatic shoulder previously (Table 1). No differences in patient characteristics were found between the total group and the patients who underwent arthroscopy.

\section{MR Arthrography findings (250 MR arthrograms)}

One hundred thirty (52\%) of $250 \mathrm{MR}$ arthrograms were performed on the $1.0 \mathrm{~T}$ unit and $120(48 \%)$ on the $1.5 \mathrm{~T}$ unit.

The MR arthrography results as scored by each individual observer for the total group of patients $(n=250)$ are shown in Table 2. All conventional MR arthrography series were considered being of sufficient quality to be evaluated by all three reviewers. Seven to nine MR arthrography series in ABER position were classified as "not evaluable." More than two thirds of supraspinatus tendons were classified as intact. Of all detected supraspinatus tendons tears, the majority was classified as type $\mathrm{C}$ lesions.

Examples of type A, B, and C lesions on T1-weighted fat suppressed TSE MR arthrography images in ABER position are shown in Figs. 2, 3, and 4.

\section{Interobserver variability}

The results of the interobserver variability are shown in Table 3, agreements ranged from $72 \%$ to $80 \%$. Weighted $\kappa$ values ranged from 0.48 up to 0.68 , interpreted as moderate to good. The weighted $\kappa$ values between the ABER and conventional MR arthrography were similar. Agreement between reviewers 1 and 2 was good, suggesting a good reproducibility by radiologists.

MR arthrography versus arthroscopy (study group, $n=92$ )

In 92 of 250 patients, an arthroscopy was performed within 12 months between MR arthrography and arthroscopy. The

Table 2 Two hundred fifty MR arthrograms: results of ABER and conventional MR arthrography obtained by each reviewer

\begin{tabular}{|c|c|c|c|c|c|}
\hline & Intact & Type A & Type B & Type C (including FT) & Not assessable \\
\hline ABER & $189 / 169 / 177$ & $18 / 19 / 4$ & $17 / 28 / 6$ & $19 / 25 / 55$ & $7 / 9 / 8$ \\
\hline Conventional MR arthrography & $192 / 176 / 202$ & $30 / 32 / 6$ & $8 / 20 / 8$ & $20 / 22 / 34$ & $0 / 0 / 0$ \\
\hline
\end{tabular}

The results of reviewer 1 are listed before the first slash, of reviewer 2 between the two slashes, and the results of reviewer 3 behind the second slash

FT full thickness 


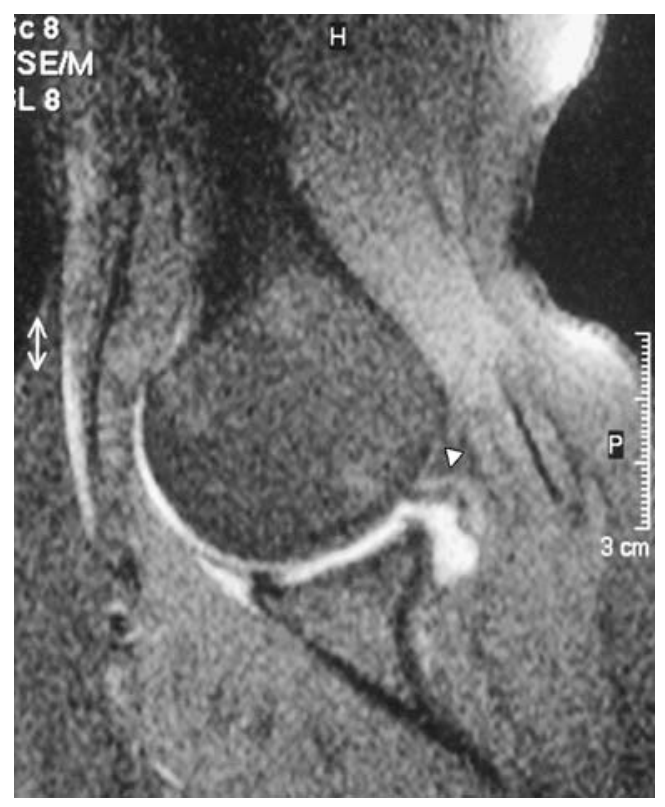

Fig. 2 Arthroscopically proven partial-thickness supraspinatus tendon tear, type A. T1-weighted fat suppressed turbo spin echo (TSE) MR arthrography image in an oblique axial plane with the arm positioned in abduction and exorotation (ABER), obtained in a 40-year-old man, shows an example of a type A horizontal partial-thickness tear (arrow) without articular surface abnormality of the supraspinatus tendon

number of patients performed on the $1.0 \mathrm{~T}$ unit and $1.5 \mathrm{~T}$ unit were equal in this study group.

According to the reference standard, 68 patients had an intact supraspinatus tendon, 17 patients had a partial thickness, and in seven patients, a full thickness lesion was detected.

The results of ABER versus conventional MR arthrography per reviewer are shown in Tables 4 and 5, respectively. Figure 5 shows an example of disagreement between ABER and conventional MR arthrography in the classification of an arthroscopically proven partial-thickness tear.

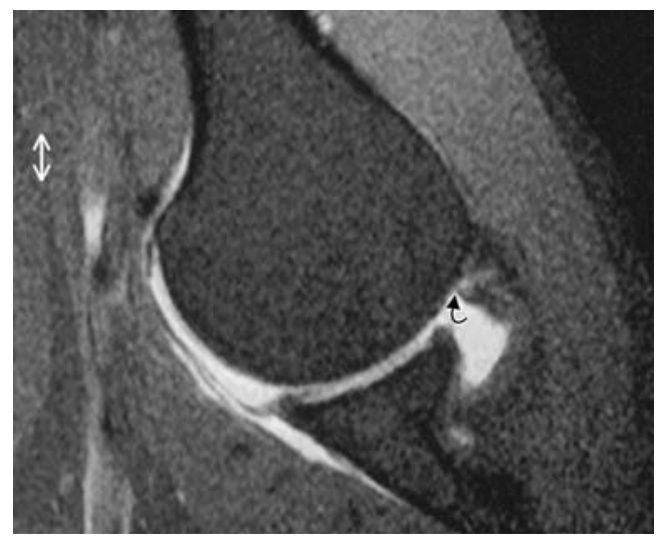

Fig. 3 Arthroscopically proven partial-thickness supraspinatus tendon tear, type B. Oblique axial T1-weighted FS TSE MR arthrography image in ABER position shows a type B horizontal partial-thickness tear with irregularity of the articular surface of the tendon near the insertion on the humerus (arrow)

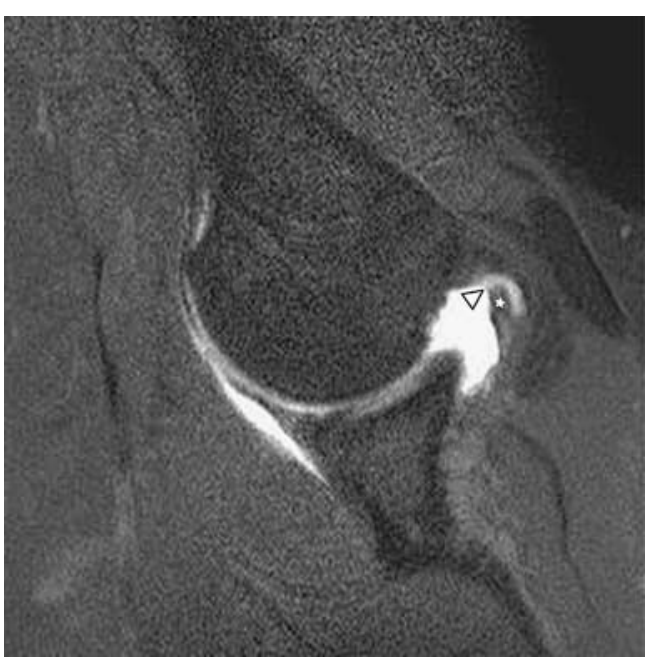

Fig. 4 Arthroscopically proven partial-thickness supraspinatus tendon tear, type $\mathrm{C}$ Oblique axial T1-weighted FS TSE MR arthrography image in ABER position, obtained in a 57-year-old man, shows a type C partial-thickness tear (arrow) with a torn edge (asterisk) of the articular surface of the tendon

\section{Sensitivity/specificity}

Sensitivity and specificity values regarding the assessment of the presence of a supraspinatus tendon tear using MR arthrography compared with arthroscopy are summarized in Table 6. Lesions not assessable on MR arthrography, if any (see Tables 4 and 5), were regarded as false negative results in the calculation of sensitivity values and as false positive results in the calculation of specificity values.

The calculated sensitivity and specificity values of ABER were 13/23 and 65/69 (reviewer 1), 11/23 and $58 / 69$ (reviewer 2), and 14/23 and 55/69 (reviewer 3). The calculated values of conventional MR arthrography were $16 / 23$ and 66/69 (reviewer 1), 15/23 and 63/69 (reviewer 2 ), and $12 / 23$ and $64 / 69$ (reviewer 3), respectively (Table 6).

No significant difference was found between the sensitivity and specificity of the conventional MR arthrography and $A B E R$.

\section{Discussion}

In the present study, we retrospectively evaluated the value of a single T1-weighted series in ABER position compared with conventional MR arthrography for the detection of supraspinatus tendon tears, with emphasis on partial tears, with arthroscopy as gold standard. Our results support the hypothesis that imaging in ABER position is as accurate as the routine series in neutral position for the detection of supraspinatus tendon tears. 
Table 3 Two hundred fifty MR arthrograms: interobserver agreement

\begin{tabular}{lccc}
\hline ABER & Percent agreement & kw value & Interpretation \\
\hline Reviewer 1 versus reviewer 2 & 80 & 0.648 & Good \\
Reviewer 1 versus reviewer 3 & 78 & 0.557 & Moderate \\
Reviewer 2 versus reviewer 3 & 72 & 0.483 & Moderate \\
Conventional MR arthrography & 79 & & \\
Reviewer 1 versus reviewer 2 & 80 & 0.675 & Good \\
Reviewer 1 versus reviewer 3 & 77 & 0.596 & Moderate \\
Reviewer 2 versus reviewer 3 & & 0.597 & Moderate \\
\hline
\end{tabular}

Ultrasonography and non-arthrographic MR imaging are accurate in the diagnosis of full-thickness rotator cuff tears with accuracy rates approaching 100\% [17-24]. Sensitivity values for the detection of partial thickness tears of both techniques, however, are less promising and are variable in literature (US, 13-80\% [15, 18, 19, 21, 25-28]; MR, 13$73 \%[18-20,22,23,25,29])$. Some recent studies prospectively compared ultrasound and conventional MR imaging with arthroscopy as reference standard: Teefey et al. found no significant difference between US and nonarthrographic MR imaging in identifying partial cuff lesions (sensitivity, 63-68\%) [18]. Martín-Hervás et al. reported higher accuracy rates of MR versus US. MR yielded sensitivity and specificity values of $50 \%$ and $76 \%$, respectively, versus $13 \%$ and $68 \%$ in US [19]. More promising results have been reported by Vlychou et al. (sensitivity, 95.6\%; specificity, 70\% [30]). The interobserver agreement on the interpretation of non-contrast MR images in partial cuff lesions is shown to be poor $k=0.13$ $0.44[22,23,31]$.

Another disadvantage of ultrasonography compared to MR imaging is the limited value in evaluation of other important structures of the shoulder like bony and labroligamentous structures. On the contrary, ultrasonography is a more cost-effective examination than MR imaging. Whereas ultrasound is operator-dependent, the interpretation of MR images is observer-dependent.

Table 4 Study group (92 MR arthrograms): results of ABER compared with arthroscopy

\begin{tabular}{lllll} 
ABER & & & & \\
\hline Intact & Type & Type & Type C & Not \\
& A & B & (including FT) & assessable \\
\hline
\end{tabular}

Arthroscopy

Intact (69)

$65 / 58 / 55 \quad 3 / 5 / 2 \quad 0 / 3 / 1 \quad 0 / 1 / 8$

$1 / 2 / 3$

Partial (16)

$7 / 8 / 8$

$2 / 1 / 1 \quad 5 / 2 / 0 \quad 1 / 3 / 7$

$1 / 2 / 0$

Full-thickness $\quad 1 / 0 / 1$

$0 / 0 / 0 \quad 0 / 0 / 0 \quad 5 / 5 / 6$

$1 / 2 / 0$

(7)

Reviewer $1 / 2 / 3$, correct correlations in italics

FT full thickness
Direct MR arthrography has been shown to be more accurate in the evaluation of the rotator cuff than conventional MR imaging and ultrasonography [20, 29, 32-34]. MR arthrography especially improves the detection of partial lesions with recently published sensitivity and specificity values of $80-95 \%$ and $96-100 \%$, respectively $[8,14,15,35]$. As mentioned previously, detection of partial thickness tears is important because even small tears can be a source of surgically treatable shoulder pain [11], and partial tears may progress to complete tears.

Indirect MR arthrography, a non-invasive examination in which intravenous administration of gadolinium provides an arthrographic effect, not needing fluoroscopic guidance and therefore not needing scheduling in two different examination rooms, might be an alternative to direct MR arthrography [36-39].

A disadvantage of indirect MR arthrography is the lack of joint distension for optimal visualization of partial-thickness articular surface tears and labroligamentous lesions.

Previous studies suggested that visualization of articular surface tears and of the horizontal component of supraspinatus tendon tears is improved by MR arthrography with additional imaging in abduction external rotation (ABER) position of the shoulder [13, 16, 39]. A disadvantage of additional imaging in ABER positioning is the prolonged scan time (approximately $15 \mathrm{~min}$ ). Therefore, the additional use of imaging in ABER position is not widespread. In an attempt to reduce scan time and to reproduce the sensitivity

Table 5 Study group (92 MR arthrograms): results of conventional MR arthrography compared with arthroscopy

Conventional MR arthrography

\begin{tabular}{lllll}
\hline Intact & Type & Type & $\begin{array}{l}\text { Type C } \\
\text { (including FT) }\end{array}$ & $\begin{array}{l}\text { Not } \\
\text { assessable }\end{array}$ \\
& $\mathrm{A}$ & $\mathrm{B}$ & &
\end{tabular}

Arthroscopy

Intact (69) $\quad 66 / 63 / 64 \quad 3 / 6 / 2 \quad 0 / 0 / 0 \quad 0 / 0 / 3 \quad 0 / 0 / 0$

$\begin{array}{llllll}\text { Partial (16) } & 7 / 8 / 11 & 6 / 4 / 1 & 2 / 2 / 1 & 1 / 2 / 3 & 0 / 0 / 0\end{array}$

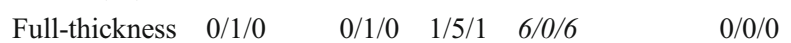

(7)

*Reviewer $1 / 2 / 3$, correct correlations in italics 

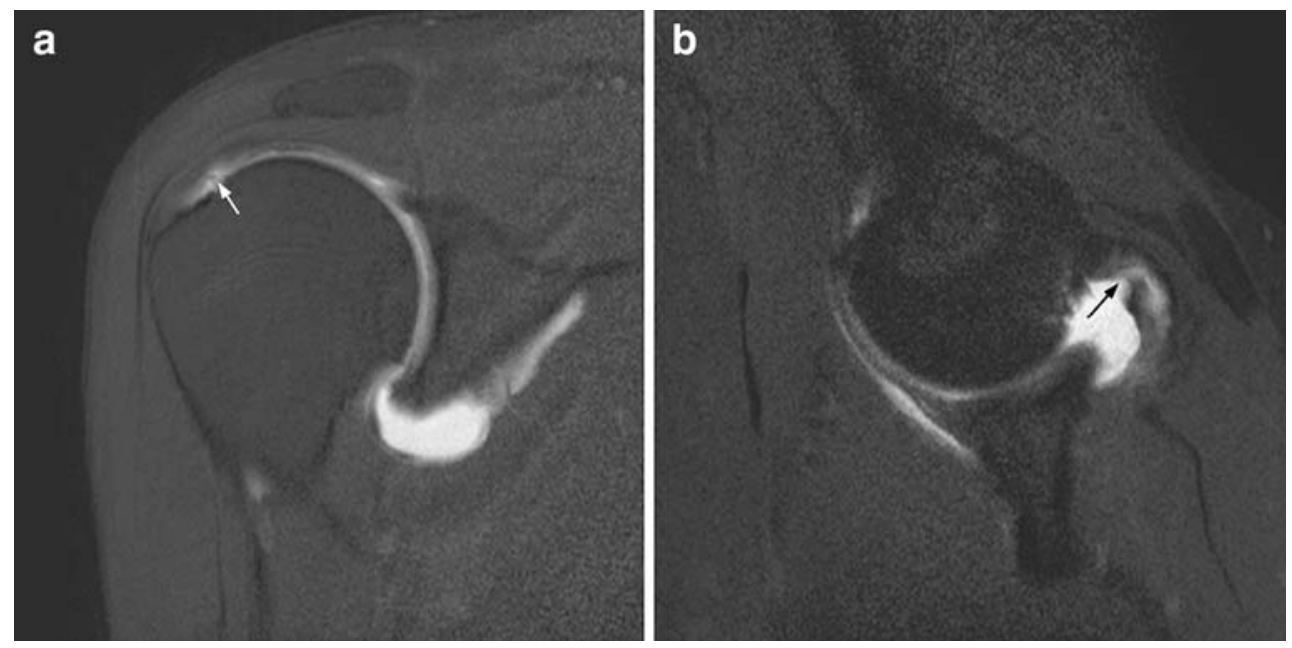

Fig. 5 Example of disagreement between ABER and conventional MR arthrography in the classification of an arthroscopically proven partial-thickness tear: a oblique coronal T1-weighted FS TSE MR arthrography image in neutral position shows subtle irregularity (arrow) of the articular surface of the supraspinatus tendon (type B).

and specificity values known from literature for the detection of partial rotator cuff tears, we investigated the value of a separate single series in ABER position. In our study, agreement between conventional MR arthrographic and arthroscopic detection was obtained with a sensitivity ranging from $52 \%$ up to $70 \%$ and a specificity ranging from $91 \%$ up to $96 \%$. No statistically significant differences were found between these results of conventional MR arthrography and a single series in ABER position (sensitivity 48$61 \%$ and specificity $80-94 \%$ ). We were not able to calculate the overall accuracy for both protocols because arthrographic (type A, B, or C) and arthroscopic (partial/full thickness) classification of cuff lesions differed. To our knowledge, our study is the first that applied the classification proposed by Lee [13]. Although moderate to high weighted $k$ values $(0.48-0.67)$ indicate an acceptable reproducibility, the value of the Lee classification in clinical practice remains uncertain.

The relatively low sensitivity values can be explained by the underestimation of small cuff lesions assessed by MR arthrography and by the gold standard "arthroscopy." For instance, according to arthroscopy, reviewer 1 incorrectly

b oblique axial T1-weighted FS TSE MR arthrography image in ABER position of the same patient shows a significant partialthickness tear of the supraspinatus tendon (arrow) with a torn edge of the articular surface (type C)

diagnosed three supraspinatus tendons as being injured on both ABER and conventional MR arthrography. All were classified as type A lesions. These lesions might have been too small or even not visible arthroscopically. Seven out of eight false negatives classified by reviewer 1 on the ABER series and all seven false negatives diagnosed on the conventional MR arthrography were arthroscopically classified as partial lesions. Thus, detecting small partial lesions on both MR and arthroscopy will remain a diagnostic challenge. On the other hand, when an appropriate amount of intra-articular contrast is applied, this series is particularly worthwhile for exclusion of partial-thickness articular surface tears.

Bursal surface tears, which are much less common than articular surface tears, are not likely to be detectable on a single T1-weighted fat-suppressed series in ABER position. To be able to detect bursal surface tears, it is crucial to include an oblique coronal either a T2-weighted spin echo, a T2-weigthed FSE, or a fat suppressed T2-weighed TSE sequence. In this study, we did not evaluate the ABER series or conventional MR arthrography series on the presence or absence of bursal surface tears.

Table 6 Study group (92 MR arthrograms): sensitivity and specificity in percentages

\begin{tabular}{|c|c|c|c|c|c|c|}
\hline & \multicolumn{2}{|c|}{ Sensitivity (\%) } & \multirow[t]{2}{*}{$p$ values } & \multicolumn{2}{|c|}{ Specificity (\%) } & \multirow[t]{2}{*}{$p$ values } \\
\hline & ABER & Conventional MR arthrography & & ABER & Conventional MR arthrography & \\
\hline Reviewer 1 & 56.5 & 69.6 & 0.38 & 94.2 & 95.5 & 1.00 \\
\hline Reviewer 2 & 47.8 & 65.2 & 0.29 & 84.0 & 91.3 & 0.27 \\
\hline Reviewer 3 & 60.9 & 52.2 & 0.69 & 79.9 & 92.8 & 0.05 \\
\hline
\end{tabular}

$p<0.05$ : significant 
In patients suffering from shoulder complaints, the cuff is not always the only source of pain. Therefore, ideally, the chosen imaging technique should allow accurate detection of other abnormalities like labroligamentous and bony lesions. Hill Sachs lesions can be detected by a single series in ABER position. In a study performed at our institution, we found that a single series in ABER position is as accurate as conventional MR arthrography series in detecting anterior labral lesions [40]. In exploring bony changes of the glenoid, however, a single series in ABER position is assumed to be of limited value. To detect and quantify bony glenoid defects, a series in sagittal plane should be added [41].

We acknowledge the known disadvantages of MR arthrography, being an invasive technique, contraindicated in patients with certain implants. Furthermore, some patients may not tolerate ABER positioning due to provocation of instability or pain. In our experience, the number of patients that could not be evaluated in ABER position does not exceed $8 \%$ of the total group of shoulder MR arthrograms.

In summary, our results show that a single MR arthrography series in ABER position and conventional MR arthrography series have equal sensitivity and specificity values when compared with arthroscopy in detecting supraspinatus tendon tears.

Acknowledgement The authors would like to thank Maartje Kunen for the illustration of type A, B, and C supraspinatus tendon tears according to the classification proposed by Lee et al. [13].

Open Access This article is distributed under the terms of the Creative Commons Attribution Noncommercial License which permits any noncommercial use, distribution, and reproduction in any medium, provided the original author(s) and source are credited.

\section{References}

1. van der Windt DA, Koes BW, de Jong BA, Bouter LM. Shoulder disorders in general practice: incidence, patient characteristics, and management. Ann Rheum Dis. 1995; 54(12): 959-964.

2. Codman EA, Akerson ID. The pathology associated with rupture of the supraspinatus tendon. Ann Surg. 1931; 93: 348.

3. Ogata S, Uhthoff HK. Acromial enthesopathy and rotator cuff tear: a radiologic and histologic postmortem investigation of the coracoacromial arch. Clin Orthop. 1990; 254: 39-48.

4. Bigliani LU, Morrison DS, April EW. The morphology of the acromion and the rotator cuff: importance. Orthop Trans. 1986; 10: 228 .

5. Soslowsky LJ, Carpenter JE, Bucchieri JS, Flatow EL. Biomechanics of the rotator cuff. Orthop Clin North Am. 1997; 28: 17-30.

6. Meister K. Injuries to the shoulder in the throwing athlete. Part I: Biomechanics/pathophysiology/classify-cation of injury. Am J Sports Med. 2000; 28: 265-275.

7. Yamaguchi K, Ditsios K, Middleton WD, Hildebolt CF, Galatz LM, Teefey SA. The demographic and morphological features of rotator cuff disease - a comparison of asymptomatic and symptomatic shoulders. J Bone Jt Surg Am. 2006; 88: 1699-1704.
8. Stetson WB, Phillips T, Deutsch A. The use of magnetic resonance arthrography to detect partial-thickness rotator cuff tears. J Bone Jnt Surg Am. 2005; 87: 81-88.

9. Ozaki J, Fujimoto S, Nakagawa Y, Masuhara K, Tamai S. Tears of the rotator cuff of the shoulder associated with pathological changes in the acromion. A study in cadavera. J Bone Jt Surg Am. 1988; 70(8): 1224-1230.

10. Budoff JE, Nirschl RP, Guidi EJ. Débridement of partial-thickness tears of the rotator cuff without acromioplasty. Long-term follow-up and review of the literature. J Bone Jt Surg Am. 1998; 80(5): 733-748.

11. Razmjou H, Holtby R, Aarabi M. Partial and full-thickness rotator cuff tears: range of motion comparison. J Bone Jt Surg Am. 2008; 90-B: 140.

12. Ellman H. Diagnosis and treatment of incomplete rotator cuff tears. Clin Orthop. 1990; 254: 64-74.

13. Lee SY, Lee JK. Horizontal component of partial-thickness tears of rotator cuff: imaging characteristics and comparison of ABER view with oblique coronal view at MR arthrography-initial results. Radiology. 2002; 224: 470-476.

14. Waldt S, Bruegel M, Mueller D, et al. Rotator cuff tears: assessment with MR arthrography in 275 patients with arthroscopic correlation. Eur Radiol. 2007; 17(2): 491-498.

15. Ferrari FS, Governi S, Burresi F, Vigni F, Stefani P. Supraspinatus tendon tears: comparison of US and MR arthrography with surgical correlation. Eur Radiol. 2002; 12(5): 1211-1217.

16. Tirman PF, Bost FW, Steinbach LS, et al. MR arthrographic depiction of tears of the rotator cuff benefit of abduction and external rotation of the arm. Radiology. 1994; 192: 851-856.

17. Steinbach LS, Palmer WE, Schweitzer ME. Special focus session: MR arthrography. RadioGraphics. 2002; 22(5): 1223-1246.

18. Teefey SA, Rubin DA, Middleton WD, Hildebolt CF, Leibold RA, Yamaguchi K. Detection and quantification of rotator cuff tears. J Bone Jt Surg Am. 2004; 86: 708-716.

19. Martín-Hervás C, Romero J, Navas-Acien A, Reboiras JJ, Munuera L. Ultrasonographic and magnetic resonance images of rotator cuff lesions compared with arthroscopy or open surgery findings. J Shoulder Elbow Surg. 2001; 10(5): 410-415.

20. Hodler J, Kursunoglu-Brahme S, Snyder SJ, Cervilla V, Karzel RP, Schweitzer ME. Rotator cuff disease: assessment with MR arthrography versus standard MR imaging in 36 patients with arthroscopic confirmation. Radiology. 1992; 182(2): 431-436.

21. Read JW, Perko M. Shoulder ultrasound: diagnostic accuracy for impingement syndrome, rotator cuff tear, and biceps tendon pathology. J Shoulder Elbow Surg. 1998; 7(3): 264-271.

22. Robertson PL, Schweitzer ME, Mitchell DG, et al. Rotator cuff disorders: interobserver and intraobserver variation in diagnosis with MR imaging. Radiology. 1995; 194: 831-835.

23. Balich SM, Sheley RC, Brown TR, Sauser DD, Quinn SF. MR imaging of the rotator cuff tendon: interobserver agreement and analysis of interpretive errors. Radiology. 1997; 204: 191-194.

24. Magee T, Williams D. 3.0-T MRI of the supraspinatus tendon. Am J Roentgenol. 2006; 187: 881-886.

25. Ianotti JP, Ciccone J, Buss DD, et al. Accuracy of office-based ultrasonography of the shoulder for the diagnosis of rotator cuff tears. J Bone Jt Surg Am. 2005; 87(6): 1305-1311.

26. Jacobson JA, Lancaster S, Prasad A, van Holsbeeck MT, Craig JG, Kolowich P. Full-thickness and partial-thickness supraspinatus tendon tears: value of US signs in diagnosis. Radiology. 2004; 230: 234-242.

27. Brenneke SL, Morgan CJ. Evaluation of ultrasonography as a diagnostic technique in the assessment of rotator cuff tendon tears. Am J Sports Med. 1992; 20: 287-289.

28. Milosavljevic J, Elvin A, Rahme H. Ultrasonography of the rotator cuff: a comparison with arthroscopy in one-hundred-andninety consecutive cases. Acta Radiol. 2005; 46(8): 858-865.

29. Flannigan B, Kursunoglu-Brahme S, Snyder S, Karzel R, Del Pizzo W, Resnick D. MR arthrography of the shoulder comparison with conventional MR imaging. AJR Am J Roentgenol. 1990; 155: 829-832. 
30. Vlychou M, Dailiana Z, Fotiadou A, Papanagiotou M, Fezoulidis IV, Malizos K. Symptomatic partial rotator cuff tears: diagnostic performance of ultrasound and magnetic resonance imaging with surgical correlation. Acta Radiol. 2009; 50(1): 101-105.

31. Middleton WD, Teefey SA, Yamaguchi K. Sonography of the rotator cuff: analysis of interobserver variability. AJR Am J Roentgenol. 2004; 183: 1465-1468.

32. Tirman PF, Palmer WE, Feller JF. MR arthrography of the shoulder. Magn Reson Imaging Clin N Am. 1997; 5: 811-839.

33. Palmer WE, Brown JH, Rosenthal DI. Rotator cuff: evaluation with fat-suppressed MR arthrography. Radiology. 1993; 188: 683-687.

34. Magee T. 3-T MRI of the shoulder: is MR arthrography necessary? Am J Roentgenol. 2009; 192: 86-92.

35. Meister K, Thesing J, Montgomery WJ, Indelicato PA, Walczak S, Fontenot W. MR arthrography of partial thickness tears of the undersurface of the rotator cuff: an arthroscopic correlation. Skelet Radiol. 2004; 33(3): 136-141.

36. Yagci B, Manisali M, Yilmaz E, Ozkan M, Ekin A, Ozaksoy D, et al. Indirect MR arthrography of the shoulder in detection of rotator cuff ruptures. Eur Radiol. 2001; 11(2): 258-262.
37. Oh DK, Yoon YC, Kwon JW, et al. Comparison of indirect isotropic MR arthrography and conventional MR arthrography of labral lesions and rotator cuff tears: a prospective study. AJR Am J Roentgenol. 2009; 192(2): 473-479.

38. Jung JY, Yoon YC, Yi SK, Yoo J, Choe BK. Comparison study of indirect MR arthrography and direct MR arthrography of the shoulder. Skeletal Radiol. 2009 (in press).

39. Herold $\mathrm{T}$, Bachthaler M, Hamer OW, et al. Indirect $\mathrm{MR}$ arthrography of the shoulder: use of abduction and external rotation to detect full- and partial-thickness tears of the supraspinatus tendon. Radiology. 2006; 240(1): 152-160.

40. Schreinemachers SA, Hulst van der VPM, Willems WJ, Bipat S, Woude van der HJ. Is a single DIRECT MR arthrography series in ABER position as accurate in detecting anteroinferior labroligamentous lesions as conventional MR arthography? Skeletal Radiol 2009 (in press).

41. Huijsmans PE, Haen PS, Kidd M, Dhert WJ, van der Hulst VP, Willems WJ. Quantification of a glenoid defect with threedimensional computed tomography and magnetic resonance imaging: a cadaveric study. J Shoulder Elbow Surg. 2007; 16(6): 803-809. 